\title{
Efficacy and Safety of Neostigmine and Decompressive Colonoscopy for Acute Colonic Pseudo-Obstruction: A Single-Center Analysis
}

\author{
Joy J. Liu ${ }^{\mathrm{a}, \mathrm{c}}$, Vishnu Venkatesh ${ }^{\mathrm{b}}$, Jing Gao ${ }^{\mathrm{b}}$, Emerald Adler ${ }^{\mathrm{a}}$, Darren M. Brenner ${ }^{\mathrm{a}}$
}

\begin{abstract}
Background: Acute colonic pseudo-obstruction (ACPO) is characterized by acute colonic dilation in the absence of anatomical obstruction. Neostigmine is an acetylcholinesterase inhibitor recommended as first-line salvage therapy for uncomplicated ACPO. Decompressive colonoscopy is recommended if neostigmine is contraindicated or unsuccessful. There is a need to better characterize relative efficacy and factors impacting treatment choice. The aim of the study was to examine the use, efficacy, and safety of neostigmine and decompressive colonoscopy in the management of ACPO at a single academic center.

Methods: Patients $\geq 18$ years of age meeting established criteria for uncomplicated ACPO and with cecal diameter $\geq 10 \mathrm{~cm}$ on imaging between 1999 and 2019 were identified. Individuals were categorized as having received supportive care alone or subsequent trials of neostigmine or decompressive colonoscopy. Demographics and pre- and post-intervention data were collected, including indication and contraindication to intervention used, time to intervention, initial response, and adverse events.
\end{abstract}

Results: In 46 cases of ACPO ( $\mathrm{N}=42$ patients), all but one individual received initial supportive care. Seven responded to conservative measures alone. Of the patients failing supportive care, 15 cases were initially treated with neostigmine (response rate $86.7 \%$ ) and 24 initially underwent decompressive colonoscopy (response rate $95.8 \%$ ) $(\mathrm{P}=0.390)$. One episode of transient bradycardia, resolved with atropine, occurred in the neostigmine group. One patient experienced respiratory instability during colonoscopy.

Conclusions: Both neostigmine and decompressive colonoscopy ap-

Manuscript submitted April 18, 2021, accepted May 3, 2021

Published online June 19, 2021

aDivision of Gastroenterology and Hepatology, Department of Internal Medicine, Northwestern University-Feinberg School of Medicine, Chicago, IL, USA

${ }^{b}$ Feinberg School of Medicine, Northwestern University, Chicago, IL, USA ${ }^{\mathrm{c} C}$ Corresponding Author: Joy Liu, Division of Gastroenterology and Hepatology, Department of Internal Medicine, Northwestern University-Feinberg School of Medicine, 676 N St Clair St, Suite 1400, Chicago, IL 60611, USA. Email: joy.liu@northwestern.edu

doi: https://doi.org/10.14740/gr1394 pear effective for treating uncomplicated ACPO in individuals failing conservative therapy. Adverse events were infrequent in both cohorts. Future prospective studies examining treatment for ACPO should focus on whether either intervention is superior to the other.

Keywords: Acute colonic pseudo-obstruction; Colonic ileus; Ogilvie syndrome; Neostigmine; Decompressive endoscopy

\section{Introduction}

Acute colonic pseudo-obstruction (ACPO) or Ogilvie's syndrome is characterized by acute dilation of the colon, usually the proximal colon, without mechanical obstruction. The incidence rates of ACPO are estimated to be approximately 1 per 1,000 patient admissions per year. ACPO is associated with conditions including recent surgery, non-operative trauma, cardiac disease, sepsis, electrolyte imbalance, and the administration of medications altering intestinal motility. It is more common in males, individuals over the age of 60 , and typically manifests as abdominal pain, distention, and alterations in bowel habits [1]. The pathophysiology of ACPO is incompletely understood but is thought to be related to disruptions in the enteric nervous system, specifically, imbalances between stimulatory neurotransmitters mediating contraction such as acetylcholine (Ach), and inhibitory neurotransmitters mediating relaxation including nitric oxide (NO) and vasoactive intestinal peptide (VIP) [1, 2]. Patients with uncomplicated ACPO (cecal diameter $<12 \mathrm{~cm}$, absence of significant abdominal pain, ischemia, or peritonitis) should initially receive conservative treatment including mobilization, electrolyte repletion, and nasogastric or rectal tube decompression. For those failing 48 to $72 \mathrm{~h}$ of conservative treatment, additional intervention is warranted [3].

In 2020, the American Society for Gastrointestinal Endoscopy (ASGE) recommended neostigmine, an acetylcholinesterase inhibitor, as a first-line treatment for ACPO when $72 \mathrm{~h}$ of supportive measures have failed and/or when the patient is not a candidate for supportive care, assuming that there is no contraindication to its use. Neostigmine reduces the degradation of acetylcholine in the enteric nervous system, stimulating peristalsis via activation of muscarinic acetylcholine receptors located on the surfaces of colonic smooth muscles cells. 
A single dose of $2 \mathrm{mg}$ given as an intravenous bolus over 3 - 5 min often causes flatus or passage of stool within hours if not minutes, with success rates reported between $88 \%$ and $94 \%$ $[4,5]$. Additional doses of neostigmine can be considered in non- or partial responders, and neostigmine infusion has been described $[6,7]$. Contraindications to neostigmine use include a known allergy to the medication, recent myocardial infarction, reactive airway disease, chronic kidney disease and uncontrolled arrhythmias. Symptomatic bradycardia may occur upon administration and for this reason, continuous cardiac monitoring and atropine availability are recommended.

Despite this recommendation, endoscopic decompression has also historically been used as an initial intervention with success rates ranging from $73 \%$ to $100 \%$ [8-12]. Factors driving colonoscopy remain unknown, although it may be presumed that this is due to the presence of a contraindication to neostigmine and/or unfamiliarity with its use. The ASGE guideline defines colonoscopy as an important alternative, although evidence to recommend it as first-line therapy is lacking, and suggests that it be used when there are absolute contraindications to neostigmine. Contraindications to endoscopic management include peritonitis or frank colonic perforation, which should prompt urgent surgical evaluation [8]. If colonoscopy is performed, placement of a decompression tube is recommended. However, more recent data from a few small retrospective studies have suggested that endoscopy may be more effective than neostigmine, and raised questions of whether it is preferrable to use neostigmine or decompressive colonoscopy as first-line therapy [3].

Given the recent ASGE-recommended preference for neostigmine and the uncertainty of next-best intervention after the failure of standard measures, the efficacy and safety of neostigmine and decompressive colonoscopy for ACPO need to be better defined. Consequently, the goals of this retrospective, single-center study were to: 1) quantify the real-world use of neostigmine and endoscopic decompression for ACPO; 2) identify factors potentially influencing treatment choice, outcomes, and rates of adverse events; and 3) provide clinical evidence of response and safety rates for neostigmine and decompressive colonoscopy.

\section{Materials and Methods}

This study was reviewed by the Institutional Review Board and deemed exempt from approval. This study was conducted in compliance with the ethical standards of the responsible institution on human subjects as well as with the Helsinki Declaration. Patients 18 years of age or older were identified for inclusion based on the International Classification of Diseases (ICD) coding systems used between 1999 and 2019 at our institution and its affiliates. The ICD codes included were: 1) ICD9 code 560.89 (other specified intestinal obstruction); 2) ICD10 code for K56.69 (other specified intestinal obstruction); 3) K59.8 (intestinal pseudo-obstruction); 4) K59.81 (Ogilvie's syndrome, ACPO); and 5) K59.89 (atony of colon, pseudo-obstruction of colon). A cecal diameter $\geq 10 \mathrm{~cm}$ was used as our minimum threshold, as this has historically been used to define ACPO [5]. Individuals were excluded from the study if cecal diameter could not be confirmed. Patients were also excluded if they were diagnosed with a mechanical bowel obstruction (small bowel obstruction, volvulus, symptomatic abdominal hernia, colonic stricture, intra-abdominal adhesions), or had a history of inflammatory bowel disease, abdominal malignancy, or prior diagnosis of chronic intestinal pseudo-obstruction (CIPO). Patients who received neostigmine during admission for reasons other than from ACPO, including neuromuscular blockade or myasthenic crisis, were also excluded.

Demographic information compiled included age, sex, and race/ethnicity as well as history of medical comorbidities (myocardial infarction, congenital heart failure, cerebral vascular accident, dementia, pulmonary disease, rheumatic disease, liver disease, type 2 diabetes mellitus, neuromuscular disease, renal disease, malignancy), recent surgical procedures (within 2 weeks prior to ACPO diagnosis), recent diagnosis of sepsis, and whether patients received opiates (acute or chronic use) prior to the onset of ACPO. We also documented whether supportive care (bowel rest, placement of nasogastric tube, electrolyte repletion, bowel regimen, and cessation of opiates) was provided prior to neostigmine or decompressive colonoscopy.

For patients who received neostigmine or underwent decompressive colonoscopy, time of diagnosis was determined by the timing of abdominal imaging and measurement of cecal diameter. The number of hours from diagnosis to intervention (either recorded administration of neostigmine or start time of colonoscopy) was determined by review of the medical record. Neostigmine dosing (both amount and number of doses administered) was recorded. Radiology reports were initially used to identify maximum cecal diameter with results reconfirmed independently by a member of the study team when images were available (54 of 73 relevant cases). When the radiology report did not explicitly state the cecal diameter and a measurement could not be confirmed, the patient was excluded.

With the exception of a single individual who did not receive supportive care as the primary intervention, episodes of ACPO were categorized by response to conservative therapy. Patients failing supportive care were then categorized based on the initial intervention (neostigmine or decompressive colonoscopy) received. This approach allowed us to account for patients who ultimately received both interventions. For individuals receiving both neostigmine and colonoscopy, the order of intervention was coded. A positive response to either intervention was determined by any one of the following criteria: 1) documentation of the passage of flatus and relief of symptoms; 2) documented clinician-determined decrease in abdominal distention; and 3) documented decrease in colonic diameter on follow-up imaging. Lack of response was defined as a lack of change of symptoms, unchanged or increased colonic distention on repeat imaging, or the need for surgical intervention to ameliorate symptoms.

For patients receiving neostigmine, objective data including blood pressure (systolic), heart rate, and serum creatinine were reviewed for the $24-\mathrm{h}$ period prior to administration. Episodes of bowel perforation, arrhythmia, hypotension, allergic reaction/anaphylaxis, bronchospasm, and cholinergic crisis that occurred after neostigmine administration were also documented. 
For patients undergoing endoscopic decompression, reports were reviewed for evidence of bowel ischemia (use of the word "ischemia" or descriptions of altered vascularity, ede$\mathrm{ma} /$ congestion and friability, or ischemic or necrotic-appearing bowel in procedure reports), placement of decompression tubes, and any other complications (including perforation and anesthesia-related adverse events). Potential contraindications to neostigmine in this group of patients including clinically suspected colonic obstruction (based on documentation), arrhythmias, hypotension, serum creatinine $>3.0$, a history of asthma exacerbation necessitating hospitalization/steroids, and severe COPD requiring home oxygen were also recorded.

We estimated based on a review of the literature that a $20 \%$ difference in response rate or efficacy (as defined above by relief in symptoms, clinician judgment, and/or improvement in imaging) would represent a clinically significant difference between the use of neostigmine or colonoscopy. The sample size required to detect a statistically significant difference assuming a power of $80 \%$ with two-sided alpha 0.05 would have been 124 . Initial calculations showed that our study was underpowered to determine statistical significance (calculated power $31 \%$ ). We used a z-test of proportions to calculate a Pvalue for one of the outcomes, whether there was a significant difference between rate of response for neostigmine and decompressive colonoscopy. Microsoft Excel (Microsoft, Redmond, WA, USA, 2011) and RStudio (RStudio, PBC, Boston, MA, USA; 2019) were used to create tables and calculate descriptive statistics.

\section{Results}

A total of 515 records in 508 patients were identified through ICD code review. Four hundred sixty-nine cases in 466 patients were excluded due to a lack of a clear underlying diagnosis, confirmed bowel obstruction, active intra-abdominal malignancy, and CIPO. After exclusion, 73 cases of ACPO in 69 patients remained. Of these, 27 cases in 27 patients were excluded due to identification of a cecal diameter $<10 \mathrm{~cm}$ radiographically or an inability to confirm a cecal diameter $\geq$ $10 \mathrm{~cm}$. This left 46 cases of ACPO in 42 unique patients to be included in this analysis (Fig. 1).

Baseline demographics were similar between groups (Table 1). Patient ages ranged from 20 to 93 years (mean 67.1 years) and $80.4 \%$ were males. Patients receiving neostigmine as their primary intervention were on average younger (56.2 years) than those who either initially underwent colonoscopy (72.2 years) or responded to supportive care ( 73.14 years). The three most common diagnoses associated with the development of ACPO included surgery within 2 weeks of diagnosis $(41.3 \%)$, a history of ischemic stroke $(39.13 \%)$, and opioid use $(36.96 \%)$. Recent surgery $(45.83 \%)$ and recent opiate use $(50 \%)$ were more common in the patients who underwent colonoscopy as their initial intervention. Fewer patients in the initial neostigmine group received opiates $(20 \%)$.

The number of patients, cases, rates of resolution with supportive care alone and subsequent neostigmine treatments or colonoscopic decompressions are shown in Figure 1. Seven cases (seven patients) of ACPO were successfully treated with supportive care alone. Of these, one responder was a patient who had previously been treated for ACPO during an earlier index hospitalization with neostigmine. Consequently, this patient's demographic data were included in both cohorts in Table 2. Twelve patients experiencing 15 total episodes of ACPO were initially treated with neostigmine (three patients had repeat index hospitalizations), and 24 patients diagnosed with ACPO (24 episodes) initially underwent decompressive colonoscopy.

A positive outcome was achieved in 13 of 15 episodes $(86.67 \%)$ in which neostigmine was the initial treatment intervention (Table 2). Nine of these cases had an appropriate response after the first dose $(60 \%)$ with three further positive outcomes occurring after a second dose in four patients. In addition, one patient from the cohort that underwent colonoscopy as the first intervention eventually received two doses of neostigmine and responded to the second dose, for a total of four positive responses in five patients who received a second dose of neostigmine. The single patient who did not respond to a second dose of neostigmine underwent an unsuccessful decompressive colonoscopy, and ultimately responded to a third trial of neostigmine (Fig. 1). In two cases, patients failing to respond to the first dose of neostigmine did not receive a second dose and successfully underwent decompressive colonoscopies. Overall, neostigmine was well tolerated as only a single patient experienced transient asymptomatic bradycardia which resolved with a single dose of atropine. No other adverse events, including heart block, asystole, bronchospasm, or bowel perforation were reported.

The average cecal diameter of patients initially receiving neostigmine was $13.3 \mathrm{~cm}$. The average number of hours between time of diagnosis and neostigmine administration was $44.86 \mathrm{~h}$ (median $20 \mathrm{~h}$ ). After neostigmine administration, there was a mean change of $3.11 \mathrm{~cm}$ in cecal diameter (median 2.3 $\mathrm{cm}$ ), although there was considerable variability in time to follow-up abdominal imaging (Table 2). Most patients received a $2 \mathrm{mg}$ intravenous (IV) bolus, with two exceptions: one patient received a $0.5 \mathrm{mg} \mathrm{IV}$ and the other a $4.5 \mathrm{mg}$ IV bolus. Both were associated with positive responses and without evidence of adverse events. None of the patients received neostigmine infusions.

There was a similarly high rate of positive response in patients who initially underwent decompressive colonoscopy, with improvement in 23 of 24 episodes $(95.8 \%)(\mathrm{P}=0.390)$. In 22 cases, patients responded after the first colonoscopy $(91.6 \%)$, and the other achieved a successful outcome after the second procedure (Table 3 ). The individual who failed to respond to a second colonoscopy ultimately responded after two doses of neostigmine (Fig. 1). Ischemic changes were identified during four of the procedures $(16.67 \%)$.

The average cecal diameter for patients undergoing colonoscopy as their initial intervention was $12.53 \mathrm{~cm}$. The average number of hours between time of diagnosis and neostigmine administration was 39.92 h (median 33 h). Post-colonoscopy, there was a mean change of $3.68 \mathrm{~cm}$ in cecal diameter (median $3.55 \mathrm{~cm}$ ), although again there was considerable variability in time to follow-up abdominal imaging. Successful placement of decompressive tubes was reported in 14 cases $(58.3 \%)$ with a lack of response noted in one patient. There was no meaning- 


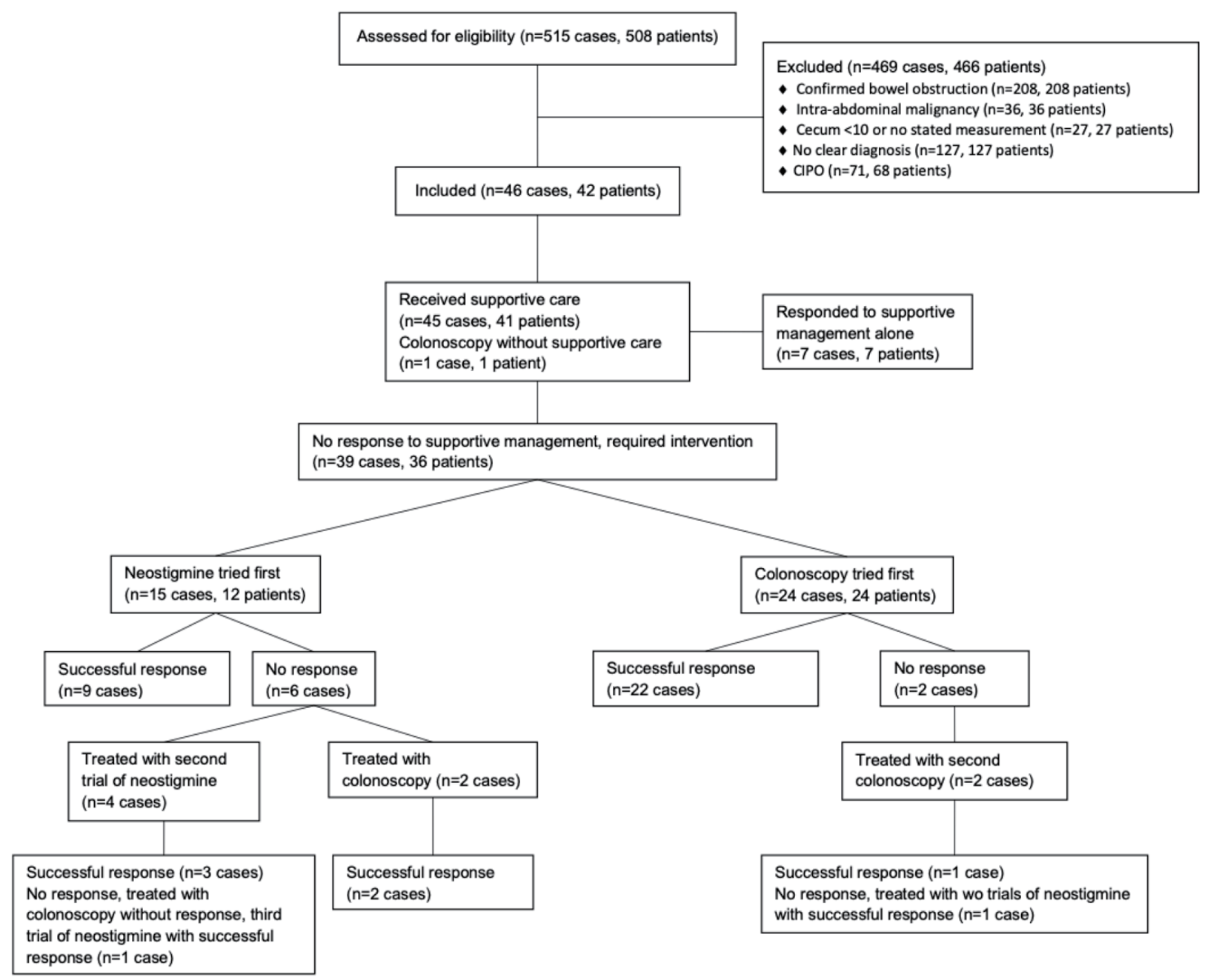

Figure 1. Patient flow and treatments for management of ACPO. A total of 515 cases in 508 patients (as some patients had repeat hospitalizations for ACPO) were initially identified. Some excluded patients did receive neostigmine or underwent colonoscopy during their hospitalization. Excluded patients receiving neostigmine: intra-abdominal malignancy $(n=1)$, cecum $<10 \mathrm{~cm}$ or no stated measurement ( $n=4$ in 4 patients), $C I P O(n=5$ in 3 patients) for a total of 10 episodes in 8 patients. Excluded patients undergoing colonoscopy: confirmed bowel obstruction ( $n=3$ in 3 patients), intra-abdominal malignancy $(n=2$ in 2 patients), cecum $<10 \mathrm{~cm}$ or no stated measurement ( $n=11$ in 11 patients), CIPO ( $n=7$ in 4 patients) for a total of 23 episodes in 20 patients. Among included cases ( $n=46$ in 42 patients), all but one patient who underwent a colonoscopy initially received supportive care. Because this patient underwent colonoscopy, they were included in the group of cases that received colonoscopy first. In the group of cases that received supportive care ( $n=45$ in 41 patients), there was one patient who responded to supportive care alone during one hospitalization and required neostigmine during a second hospitalization. This patient and their demographic information at time of hospitalization was included in both the group of cases receiving neostigmine first as well as in the group of cases responding to supportive care alone. ACPO: acute colonic pseudo-obstruction.

ful difference in the mean change in cecal diameter based on placement of a decompressive tube (placement: $3.78 \mathrm{~cm}$, nonplacement: $3.70 \mathrm{~cm}$ ) (Table 3).

Potential contraindications to neostigmine were documented in $16(67 \%)$ cases where colonoscopy was performed first. Cited contraindications included the following: clinical concern for bowel obstruction $(\mathrm{n}=6)$, history of arrhythmia $(\mathrm{n}=7)$, history of renal disease $(\mathrm{n}=1)$, history of bronchospastic disease $(n=1)$, and other $(n=1)$. Fourteen of these 16 patients had a positive response to colonoscopy (87.5\%). The other eight patients, who did not have an identifiable contrain- dication to neostigmine, but still underwent colonoscopy initially, all responded to colonoscopy and did not require further intervention.

The occurrence of adverse events was rare among patients who underwent colonoscopy first. A single bowel perforation occurred in a terminally ill patient 2 weeks after the index colonoscopy. This was not considered related to the procedure. One colonoscopy was aborted due to patient instability (hypoxia and increased abdominal pain without perforation) but this procedure was considered complete and a decompression tube was successfully placed. The patient was ultimately de- 
Table 1. Patient Characteristics

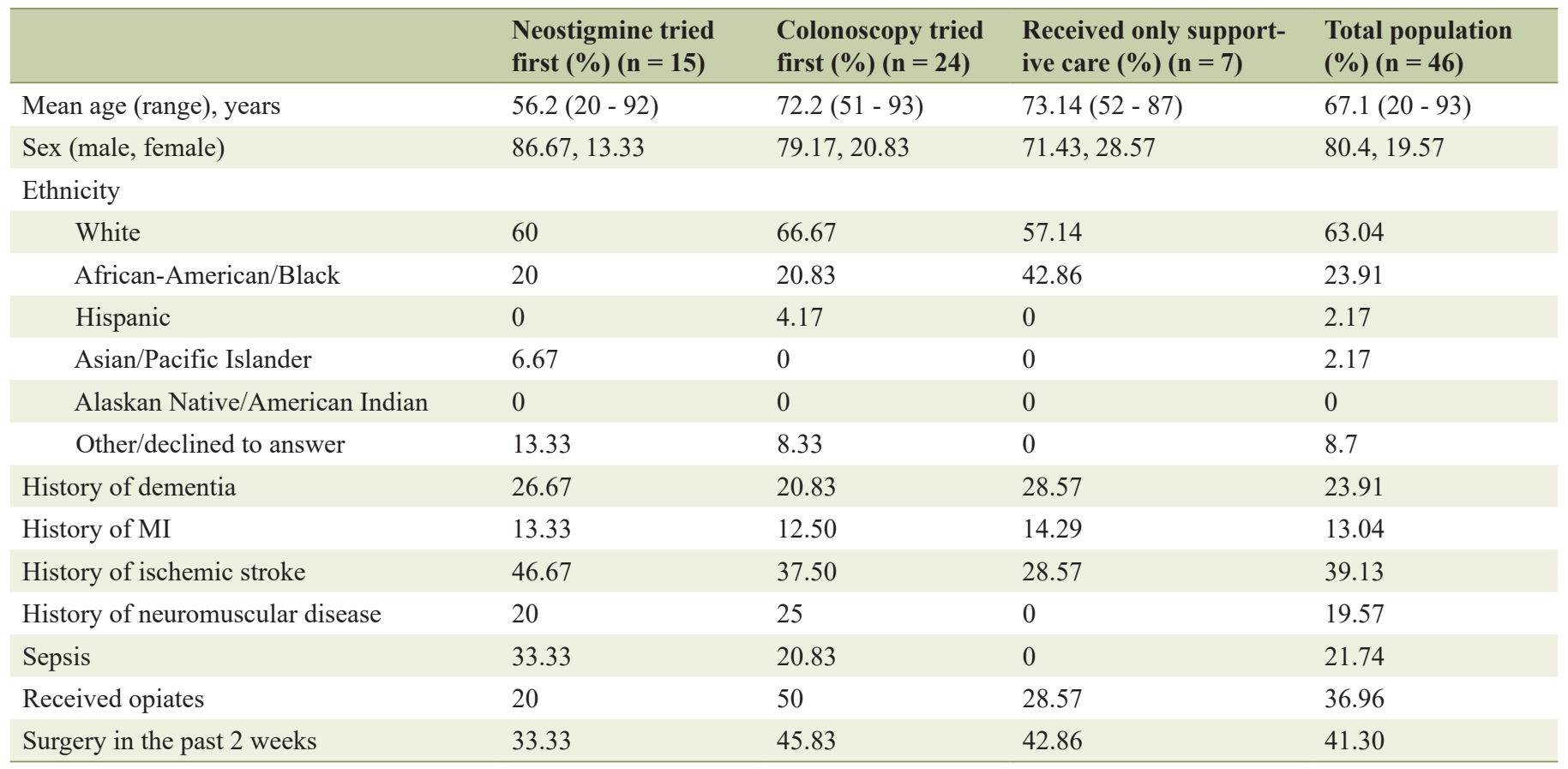

MI: myocardial infarction.

termined to have a positive response to intervention (Table 3).

\section{Discussion}

The purpose of this study was to assess the efficacy and safety of neostigmine and decompressive colonoscopy for treating $\mathrm{ACPO}$ at a single tertiary center. There were relatively few patients who responded to supportive care alone, indicating that subsequent interventions proven effective and safe are likely to be necessary. Both neostigmine and decompressive colonoscopy were effective in the majority of cases with overall response rates of $86.67 \%$ and $95.8 \%$, respectively. Both were also safe, with only a single episode of transient asymptomatic bradycardia occurring in a patient who received neostigmine, and one bowel perforation occurring 2 weeks after a successful colonoscopy. No significant differences could be discerned between initial responses to neostigmine or colonoscopy, due to the underpowered nature of our retrospective study $(\mathrm{P}=0.390)$.

Although this was a single-center study, our rates of response were similar to those reported in the general literature for both neostigmine and colonoscopy $[5,6,13]$. Patients who received a single dose of neostigmine had an approximate $60 \%$ response rate, but this rose by an additional $80 \%$ with the second dose, which is consistent with other studies in which a second bolus of neostigmine has been associated with response

Table 2. Use and Efficacy of Initial Neostigmine Trial in ACPO ( $N=15$ Episodes in 12 Patients)

\begin{tabular}{ll}
\hline Total rate of positive response $(\%)^{\mathrm{a}}$ & $13 / 15(86.67 \%)$ \\
Rate of positive response after first dose & $9 / 15(60 \%)$ \\
Rate of positive response after second dose & $4 / 5(80 \%)^{\mathrm{b}}$ \\
Number of hours between imaging findings and intervention & Median $20 \mathrm{~h}$, average $44.86 \mathrm{~h}$ \\
Average cecal diameter at time of diagnosis $(\mathrm{cm})$ & 13.3 \\
Average change in cecal diameter in responders $(\mathrm{cm})$ & $3.11($ median 2.3$)$ \\
Range of dose & $0.5-4.5$ mg IV bolus \\
Rate of positive response to colonoscopy (performed after neostigmine failed) & $3 / 3$ \\
Surgery required for ischemia or perforation & $0 / 15^{\mathrm{c}}$ \\
\hline
\end{tabular}

aPositive response was defined as documentation of passage of flatus, relief of symptoms, decrease in abdominal distention, or decrease in colon diameter on imaging. ${ }^{b}$ One patient had a positive response after receiving neostigmine three times. One patient who initially underwent colonoscopy ultimately had a response to two doses of neostigmine. 'One patient eventually underwent an elective surgery for persistent symptoms. ACPO: acute colonic pseudo-obstruction; IV: intravenous. 
Table 3. Use and Efficacy of Initial Trial of Decompressive Colonoscopy in ACPO ( $N=24$ Episodes in 24 Patients)

\begin{tabular}{ll}
\hline Total rate of positive response $(\%)^{\mathrm{a}}$ & $23 / 24(95.8 \%)$ \\
Rate of positive response after first colonoscopy (\%) & $22 / 24(91.6 \%)$ \\
Number of hours between imaging findings and intervention & Mean $39.92 \mathrm{~h}$, median $33 \mathrm{~h}$ \\
\hline Average cecal diameter at time of diagnosis $(\mathrm{cm})$ & 12.53 \\
Mean change in cecal diameter in responders (cm) & $3.68($ median 3.55) \\
Ischemia present at time of scope & $4 / 24(16.67 \%)$ \\
Obstruction present at time of scope & $0 / 24$ \\
Peri-procedure complications $(\%)$ & $1 / 24(4.17 \%)^{\mathrm{b}}$ \\
Rate of bowel perforation $(\%)$ & $0 / 24^{\mathrm{c}}$ \\
Surgery required for persistent symptoms (\%) & $1 / 24(4.17 \%)$ \\
Patients in whom neostigmine was considered contraindicated & $16 / 24(67 \%)$ \\
\hline
\end{tabular}

aPositive response was defined as documentation of passage of flatus, relief of symptoms, decrease in abdominal distention, or decrease in colon diameter on imaging. ${ }^{b}$ In one episode, hypoxia occurred during colonoscopy and although the procedure was aborted, a decompression tube was placed and the colonoscopy was considered complete. 'Bowel perforation occurred in one patient who underwent colonoscopy, 2 weeks after the procedure. ACPO: acute colonic pseudo-obstruction.

rates between $40 \%$ and $100 \%$ [3]. One patient who did not respond to a single dose of neostigmine underwent successful decompressive colonoscopy, but additional doses should be considered for patients with initially incomplete responses. All of our patients received a slow bolus of neostigmine; none received a continuous infusion which has been reported to be successful elsewhere $[6,7]$.

A review of our patient population revealed that the primary motivation for using decompressive colonoscopy as initial therapy was concern regarding the safety of neostigmine. Some of these concerns may be unfounded. One-third of the patients who initially underwent colonoscopy could have received neostigmine, as we were unable to identify any absolute contraindications. Other cited concerns such as controlled atrial fibrillation $(\mathrm{N}=6)$ and heart failure $(\mathrm{N}=1)$ also should not have precluded neostigmine use. This may be indicative of an incorrect assumption that significant cardiac disease of any kind represents an absolute contraindication to the use of neostigmine [4]. Of the 15 episodes in which neostigmine was trialed first, one case of transient bradycardia occurred with no lasting sequelae. Concerns regarding the safety of neostigmine in ACPO often limit its use, but we did not identify any serious lasting adverse effects $[10,14,15]$. These findings suggest an opportunity for further education to increase appropriate utilization of neostigmine.

Possible mechanical obstruction was the second-most commonly cited contraindication to using neostigmine in our study. Mechanical obstruction is often, but not always, ruled out via imaging, and as such, we rely on clinical judgement to determine whether neostigmine administration is appropriate. Historically the use of water-soluble contrast enemas has been advocated to rule out mechanical obstruction prior to neostigmine; however, this was not seen in our study as patients were assessed via computed tomography scan to evaluate colonic diameter and to rule out obstruction [5].

Despite the potential under-utilization of neostigmine at our institution, we found that colonoscopy was both highly effective and safe. Current ASGE practice guidelines assert that decompressive colonoscopy should be limited to patients who fail to respond to supportive care or medical management (and in whom neostigmine is contraindicated), but all eight patients who theoretically could have received neostigmine first instead underwent colonoscopy and had positive responses. In addition, colonoscopy allowed for the identification of colonic ischemia in four of 24 patients, which can be a useful prognostic finding. Recent ASGE guidelines cite two small studies arguing that decompressive colonoscopy may be superior to neostigmine with further data necessary to support this assumption $[9,10]$. Unfortunately, our study was underpowered to further validate this assertion.

The risks of complications are higher when colonoscopies are performed for ACPO as opposed to other indications. Overall, the estimated risk of bowel perforation is approximately $2 \%$ and mortality $1 \%$ [16]. In this study, a single bowel perforation was recorded, but documentation revealed that it occurred 2 weeks after the colonoscopy was completed for $\mathrm{ACPO}$, and it was not deemed related to the index procedure. One colonoscopy was aborted due to patient instability during the procedure. Overall, these data suggest that decompressive colonoscopy is safe in a population of individuals with ACPO.

There are multiple strengths to our study including the strict inclusion and exclusion criteria utilized. The requirement of a cecal diameter $\geq 10 \mathrm{~cm}$ on imaging mirrors the criterion for uncomplicated ACPO set forth in the most recent ASGE guidelines, and this requirement makes our results more clinically relevant, as the decision to intervene with either neostigmine or colonoscopy is more pressing as colon diameter increases. Furthermore, despite being a retrospective, uncontrolled analysis, our study is unique in its examination of the use of both neostigmine and colonoscopy and in its review of the rationale for choosing one intervention over the other. Our study presents granular details such as the number of doses of neostigmine or decompressive colonoscopies required to achieve a clinical response, follow-up and response to secondary interventions, and the effectiveness of decompression tube placement. These results have clinical implications for realworld outcomes. Finally, safety comparisons were performed 
for both interventions revealing that complication rates were low and similar between interventions, and neither was directly associated with serious adverse events.

There are also limitations to this study. The overall size of the population was small, and the data captured were limited to the inpatient population of a single academic center. Given our strict inclusion criteria, we excluded 27 episodes of presumed ACPO, and our study was underpowered to calculate statistical significance in outcomes between patients undergoing neostigmine or colonoscopy as initial therapy. That is to say, the data represent the totality of ACPO treatment spanning two decades at a tertiary institution. Due to the retrospective nature of our study, only data available in the electronic medical record could be analyzed. In particular, this limited our ability to understand why a gastroenterologist might choose endoscopic decompression over neostigmine when no absolute contraindications were documented. Future studies comparing these two therapies should examine factors influencing provider decision-making. Finally, the initial pool of patients was identified using ICD-9 and ICD-10 codes, so there is the possibility that some cases may have been missed.

Overall, our findings suggest that both neostigmine and decompressive colonoscopy represent effective and safe treatments for ACPO. Neostigmine may be underutilized due to concerns about safety despite very rare and often transient adverse effects. What remain to be elucidated in future large prospective randomized trials are factors predictive of positive responses or lack thereof to either neostigmine or colonoscopy and whether one of these therapeutic modalities is more effective that the other after conservative measures have failed, taking into account relative and absolute contraindications to either procedure $[5,16]$.

\section{Acknowledgments}

This work was supported in part by the Northwestern Medicine Enterprise Data Warehouse.

\section{Financial Disclosure}

None to declare.

\section{Conflict of Interest}

None to declare.

\section{Informed Consent}

Not applicable.

\section{Author Contributions}

Joy J. Liu designed the study, contributed to data collection and analysis, and drafting and critical revision of the manuscript. Vishnu Venkatesh contributed to data collection and drafting of the manuscript. Jing Gao contributed to data collection and drafting of the manuscript. Emerald Adler assisted with data analysis and critical revision of the manuscript. Darren $\mathrm{M}$. Brenner supervised the study and contributed to critical revision of the manuscript.

\section{Data Availability}

The authors declare that data supporting the findings of this study are available within the article.

\section{Abbreviations}

ACPO: acute colonic pseudo-obstruction; VIP: vasoactive intestinal peptide; ASGE: American Society for Gastrointestinal Endoscopy; CIPO: chronic intestinal pseudo-obstruction; COPD: chronic obstructive pulmonary disease; ICD: International Classification of Diseases; IV: intravenous

\section{References}

1. Vanek VW, Al-Salti M. Acute pseudo-obstruction of the colon (Ogilvie's syndrome). An analysis of 400 cases. Dis Colon Rectum. 1986;29(3):203-210.

2. Bernardi MP, Warrier S, Lynch AC, Heriot AG. Acute and chronic pseudo-obstruction: a current update. ANZ J Surg. 2015;85(10):709-714.

3. Naveed M, Jamil LH, Fujii-Lau LL, Al-Haddad M, Buxbaum JL, Fishman DS, Jue TL, et al. American Society for Gastrointestinal Endoscopy guideline on the role of endoscopy in the management of acute colonic pseudoobstruction and colonic volvulus. Gastrointest Endosc. 2020;91(2):228-235.

4. Loftus CG, Harewood GC, Baron TH. Assessment of predictors of response to neostigmine for acute colonic pseudo-obstruction. Am J Gastroenterol. 2002;97(12):31183122.

5. Ponec RJ, Saunders MD, Kimmey MB. Neostigmine for the treatment of acute colonic pseudo-obstruction. N Engl J Med. 1999;341(3):137-141.

6. White L, Sandhu G. Continuous neostigmine infusion versus bolus neostigmine in refractory Ogilvie syndrome. Am J Emerg Med. 2011;29(5):576 e571-573.

7. Smedley LW, Foster DB, Barthol CA, Hall R, Gutierrez GC. Safety and efficacy of intermittent bolus and continuous infusion neostigmine for acute colonic pseudo-obstruction. J Intensive Care Med. 2020;35(10):1039-1043.

8. Jetmore AB, Timmcke AE, Gathright JB, Jr., Hicks TC, Ray JE, Baker JW. Ogilvie's syndrome: colonoscopic decompression and analysis of predisposing factors. Dis Colon Rectum. 1992;35(12):1135-1142.

9. Tsirline VB, Zemlyak AY, Avery MJ, Colavita PD, Christmas AB, Heniford BT, Sing RF. Colonoscopy is superior 
to neostigmine in the treatment of Ogilvie's syndrome. Am J Surg. 2012;204(6):849-855; discussion 855.

10. Peker KD, Cikot M, Bozkurt MA, Ilhan B, Kankaya B, Binboga S, Seyit H, et al. Colonoscopic decompression should be used before neostigmine in the treatment of Ogilvie's syndrome. Eur J Trauma Emerg Surg. 2017;43(4):557-566.

11. Committee ASoP, Harrison ME, Anderson MA, Appalaneni V, Banerjee S, Ben-Menachem T, Cash BD, et al. The role of endoscopy in the management of patients with known and suspected colonic obstruction and pseudo-obstruction. Gastrointest Endosc. 2010;71(4):669-679.

12. Geller A, Petersen BT, Gostout CJ. Endoscopic decom- pression for acute colonic pseudo-obstruction. Gastrointest Endosc. 1996;44(2):144-150.

13. Ilban O, Cicekci F, Celik JB, Bas MA, Duman A. Neostigmine treatment protocols applied in acute colonic pseudo-obstruction disease: A retrospective comparative study. Turk J Gastroenterol. 2019;30(3):228-233.

14. Rex DK. Acute colonic pseudo-obstruction (Ogilvie's syndrome). Gastroenterologist. 1994;2(3):233-238.

15. Saunders MD. Management of colonic pseudo-obstruction. Gastroenterol Hepatol (N Y). 2006;2(12):886-887.

16. Jain A, Vargas HD. Advances and challenges in the management of acute colonic pseudo-obstruction (ogilvie syndrome). Clin Colon Rectal Surg. 2012;25(1):37-45. 